\title{
Metastatic Angiosarcoma with Kasabach-Merritt Syndrome Responsive to Gemcitabine and Vinorelbine after Failure of Liposomal Doxorubicin and Paclitaxel: A Case Report
}

\author{
William L. Read Felicia Williams \\ Winship Cancer Institute, Emory University, Atlanta, Ga., USA
}

\section{Key Words}

Angiosarcoma · Kasabach-Merritt syndrome · Chemotherapy · Thrombocytopenia

\begin{abstract}
Kasabach-Merritt syndrome (KMS) describes a consumptive coagulopathy associated with certain vascular tumors. It is thought that platelets are destroyed as they circulate through the aberrant endothelial surfaces associated with these tumors. Most published literature describes infants with kaposiform hemangioendothelioma, but a similar syndrome can complicate angiosarcoma in adults. This report describes a man with metastatic angiosarcoma arising in the scalp in whom disease progression was complicated by profound thrombocytopenia consistent with KMS. His disease and associated KMS had progressed previously through paclitaxel and then through liposomal doxorubicin. It did not respond to paclitaxel and bevacizumab, but responded almost completely to chemotherapy with gemcitabine and vinorelbine. Six months later, progression through ongoing chemotherapy then responded to chemotherapy with cyclophosphamide and sirolimus.

(C) 2016 The Author(s)

Published by S. Karger AG, Basel
\end{abstract}

Kasabach-Merritt syndrome (KMS) describes a consumptive coagulopathy associated with certain vascular tumors. It is thought that platelets are destroyed as they circulate through the aberrant endothelial surfaces associated with these tumors. Most published literature describes infants with kaposiform hemangioendothelioma [1], but a similar syndrome can complicate angiosarcoma in adults. There are several published reports of adults

\section{KARGER}

William L. Read, MD

Winship Cancer Institute, Emory University

550 Peachtree Street, MOT 18

Atlanta, GA 30308 (USA)

E-Mail wread@emory.edu 
Read and Williams: Metastatic Angiosarcoma with Kasabach-Merritt Syndrome Responsive to Gemcitabine and Vinorelbine after Failure of Liposomal Doxorubicin and Paclitaxel: A Case Report

with angiosarcoma and KMS. In most reports, the patient dies rapidly without treatment or despite attempted treatment, although there are 2 reports describing longer survival after paclitaxel $[2,3]$. The dismal outcomes of reported cases might lead oncologists to feel nihilistic about the prospect of helping a patient with angiosarcoma and KMS.

This report describes a man with metastatic angiosarcoma arising in the scalp, in whom disease progression was complicated by profound thrombocytopenia consistent with KMS. His disease and associated KMS had progressed previously through paclitaxel and then through liposomal doxorubicin. It did not respond to paclitaxel and bevacizumab, but responded almost completely to chemotherapy with gemcitabine and vinorelbine. Six months later, progression through ongoing chemotherapy then responded to chemotherapy with cyclophosphamide and sirolimus.

A 46-year-old man with no prior medical history presented with a scalp mass in 2002, which on biopsy was found to be angiosarcoma. This was treated with resection and radiation. Surveillance imaging in 2004 discovered lung lesions which were proven to be angiosarcoma on needle biopsy. He was treated with combination of paclitaxel and doxorubicin $x$ 6 cycles, with complete response. This was sustained through 2012 when he developed a recurrence in the scalp at some distance from his primary site, together with reappearance of lung and bone lesions. He once again received paclitaxel, this time weekly. This produced a response, and he continued with this for 6 cycles. Shortly after discontinuing chemotherapy, he had progression in neck adenopathy, which was confirmed on fine needle aspiration and treated with radiation in October 2012. Paclitaxel was restarted for progression in the scalp and metastatic sites but discontinued in January 2013 for continued progression. Pazopanib was attempted for 1 month but discontinued because of profound malaise and hypertension. Liposomal doxorubicin was begun in February 2013 with response in the scalp and metastatic sites, and continued through September 2013.

Platelet counts were noted to be falling in August 2013, and the patient began to complain of worsening back pain. Red and white cell lines were not affected, nor did the peripheral smear show nucleated red cells, teardrop cells or immature white cells as would be seen on a myelophthisic or leukoerythroblastic picture, with angiosarcoma filling marrow spaces. There were no schistocytes as would be seen in microangiopathic hemolytic anemia. Disseminated intravascular coagulation was considered, but prothrombin time and partial thromboplastin time remained normal, and fibrinogen was actually elevated. Platelet transfusion failed to increment the platelet count, which fell into the single digits. Despite this, the patient suffered only a slight increase in bleeding (from his scalp tumor) and bruising. These facts seemed inconsistent with a primary marrow problem, in which bleeding is more problematic and platelet transfusions do increment platelet counts. They were more consistent with a consumptive thrombocytopenia like idiopathic thrombocytopenic purpura.

After a PET scan done in September 2013 showed widespread progression of tumor in lungs and bones (fig. 1a), he was diagnosed with thrombocytopenia from KMS. Liposomal doxorubicin was discontinued. His profoundly low platelet count made chemotherapy a risky prospect, but his performance status remained good. In the thought that KMS might be mediated by angiogenic factors, paclitaxel was started again, this time with bevacizumab together with $20 \mathrm{mg}$ of dexamethasone each dose, this as described in a clinical trial for angiosarcoma ongoing at the time [4]. A total of three doses were given, with no change in platelet count and evidence of progression on early interval PET scan in October 2013 (fig. $1 b)$.

Chemotherapy was changed to vinorelbine and gemcitabine, given weekly. This regimen was described in a phase II trial for sarcoma by Dileo et al. [5], but I use the doses described 
Read and Williams: Metastatic Angiosarcoma with Kasabach-Merritt Syndrome Responsive to Gemcitabine and Vinorelbine after Failure of Liposomal Doxorubicin and Paclitaxel: A Case Report

by Bartlett et al. [6] in a clinical trial of these agents for relapsed Hodgkin disease. The threedrug GVD mix (gemcitabine $800 \mathrm{mg} / \mathrm{m}^{2} /$ week, vinorelbine $15 \mathrm{mg} / \mathrm{m}^{2} /$ week and liposomal doxorubicin $10 \mathrm{mg} / \mathrm{m}^{2} /$ week) was intended to have maximal sustainability and minimal long-term toxicity for patients with Hodgkin disease. In my practice, it has been useful for patients with angiosarcoma and leiomyosarcoma. Because this patient had just progressed through liposomal doxorubicin, this drug was omitted. He received chemotherapy weekly, initially with no weeks off.

This regimen produced a prompt normalization of platelet count and resolution of pain (fig. 3). Repeat PET scan (fig. 1c) showed resolution of all metastatic sites, although interestingly his scalp recurrence remained FDG avid. Feeling better, the patient took a 3-week holiday from chemotherapy for a previously scheduled vacation. On return, his platelet count had again fallen to the single digits, though this again recovered with restarting chemotherapy, now given every other week.

After 6 months, the falling platelet count and worsening bone pain made clear that the disease was progressing despite ongoing chemotherapy. In addition, he developed visible progression and bleeding of his scalp tumor. Progression of metastatic sites was confirmed on PET. Gemcitabine and vinorelbine was discontinued, and the patient began oral cyclophosphamide and sirolimus as described by Schuetze et al. [7]. This regimen had not helped 2 prior patients with progressive angiosarcoma in this practice but was chosen because sirolimus had been reported to have activity against the pediatric version of KMS [8] and also for lack of good alternatives. In fact, shortly after beginning the regimen, his platelet count did improve (fig. 3). Interestingly, his scalp tumor also regressed dramatically (fig. 2). This tumor had not changed much during prior chemotherapies and had remained PET avid even after all sites of metastatic disease had disappeared on PET (fig. 1). A follow-up PET scan showed that the scalp tumor was no longer PET avid. The effect against metastatic disease was less dramatic, though here too he had a response. In retrospect, it turned out that the patient was taking both cyclophosphamide and sirolimus every other week as opposed to the regimen described by Schuetze's group which entails continuous sirolimus with cyclophosphamide as the only alternating component. It is possible that the regimen might have worked better with uninterrupted sirolimus.

The patient ultimately progressed with profound thrombocytopenia (but not clinically significant bleeding). Pleural effusion was found to contain malignant cells, and bone marrow biopsy done shortly before his death confirmed a hypercellular marrow, with $30 \%$ involvement by malignant cells consistent with angiosarcoma.

This report is submitted in the hope that it might offer ideas and optimism for oncologists treating persons with metastatic angiosarcoma. Angiosarcoma can behave aggressively and is capable of rapid progression with life-threatening consequences. It is also chemoresponsive, and moderate doses of chemotherapy can produce responses, palliate symptoms and improve quality of life. The patient described here lived with metastatic disease for over 10 years, including his 2.5 final years on chemotherapy, nearly 1 year of which was after his diagnosis with KMS.

\section{Statement of Ethics}

The authors have no ethical conflicts to disclose. 
Read and Williams: Metastatic Angiosarcoma with Kasabach-Merritt Syndrome Responsive to Gemcitabine and Vinorelbine after Failure of Liposomal Doxorubicin and Paclitaxel: A Case Report

\section{Disclosure Statement}

The authors declare that they have no conflicts of interest.

\section{References}

1 O'Rafferty C, O'Regan GM, Irvine AD, et al: Recent advances in the pathobiology and management of Kasabach-Merritt phenomenon. Br J Haematol 2015;171:38-51.

2 Grellety T, Italiano A: Angiosarcoma associated with a Kasabach-Merritt syndrome: report of two cases treated with paclitaxel. Future Oncol 2013;9:1397-1399.

-3 Imafuku S, Hosokawa C, Moroi Y, et al: Kasabach-Merritt syndrome associated with angiosarcoma of the scalp successfully treated with chemoradiotherapy. Acta Derm Venereol 2008;88:193-194.

4 Ray-Coquard IL, Domont J, Tresch-Bruneel E, et al: Paclitaxel given once per week with or without bevacizumab in patients with advanced angiosarcoma: a randomized phase II trial. J Clin Oncol 2015;33:2797-2802.

5 Dileo P, Morgan JA, Zahrieh D, et al: Gemcitabine and vinorelbine combination chemotherapy for patients with advanced soft tissue sarcomas: results of a phase II trial. Cancer 2007;109:1863-1869.

6 Bartlett NL, Niedzwiecki D, Johnson JL, et al: Gemcitabine, vinorelbine, and pegylated liposomal doxorubicin (GVD), a salvage regimen in relapsed Hodgkin's lymphoma: CALGB 59804. Ann Oncol 2007;18:1071-1079.

7 Schuetze SM, Zhao L, Chugh R, et al: Results of a phase II study of sirolimus and cyclophosphamide in patients with advanced sarcoma. Eur J Cancer 2012;48:1347-1353.

8 Wang Z, Li K, Dong K, et al: Refractory Kasabach-Merritt phenomenon successfully treated with sirolimus, and a mini-review of the published work. J Dermatol 2015;42:401-404.

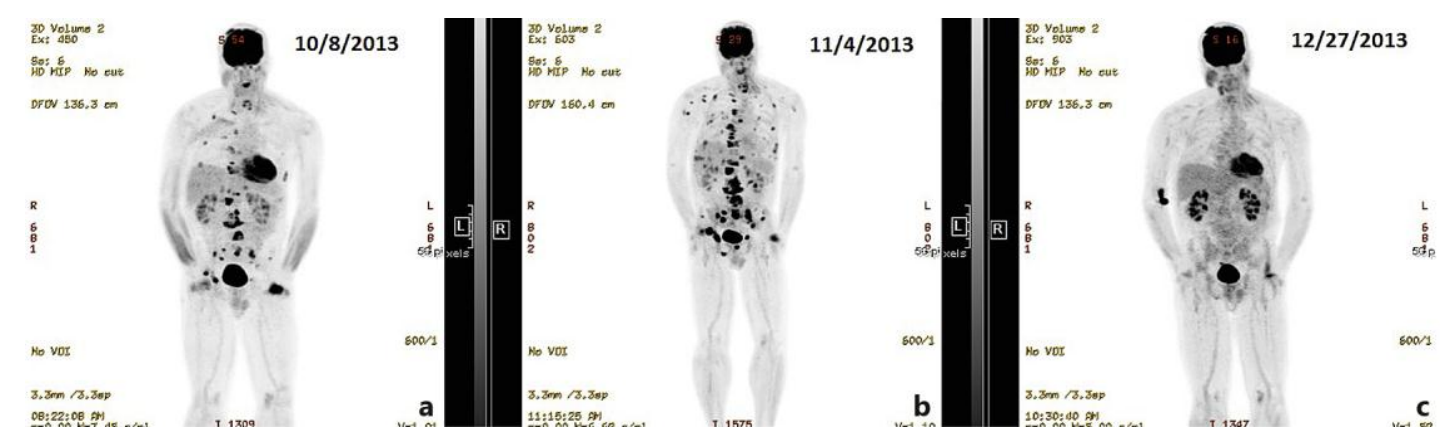

Fig. 1. PET scans. a Multiple sites of standardized uptake value (SUV) avidity can be seen in the lungs and bones, especially the spine. SUV avidity at the site of the scalp lesion is also evident. b After 1 cycle of paclitaxel and bevacizumab, the PET scan shows an increase in the number and SUV avidity of lesions consistent with progression. c After 6 weeks of gemcitabine and vinorelbine, there is no remaining SUV avidity at prior metastatic sites. The scalp lesion remains SUV avid. 


\section{Case Reports in Oncology}

\begin{tabular}{l|l}
\hline Case Rep Oncol 2016;9:177-181 \\
\hline DOI: 10.1159/000444747 & $\begin{array}{l}\text { ( ) 2016 The Author(s). Published by S. Karger AG, Basel } \\
\text { www.karger.com/cro }\end{array}$ \\
\hline
\end{tabular}

Read and Williams: Metastatic Angiosarcoma with Kasabach-Merritt Syndrome Responsive to Gemcitabine and Vinorelbine after Failure of Liposomal Doxorubicin and Paclitaxel: A Case Report
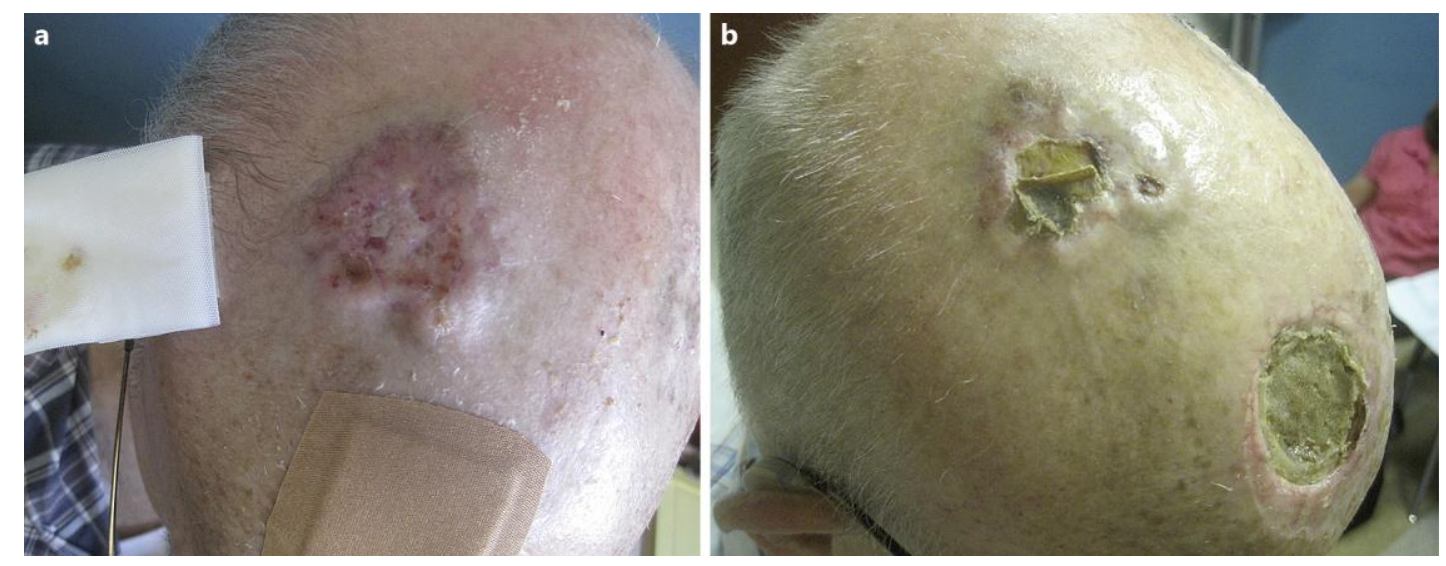

Fig. 2. Photographs of scalp recurrence. a Progression of scalp recurrence, March 2013. b Response of scalp recurrence, July 2013. The site of his original primary tumor can be seen in the anterior scalp.

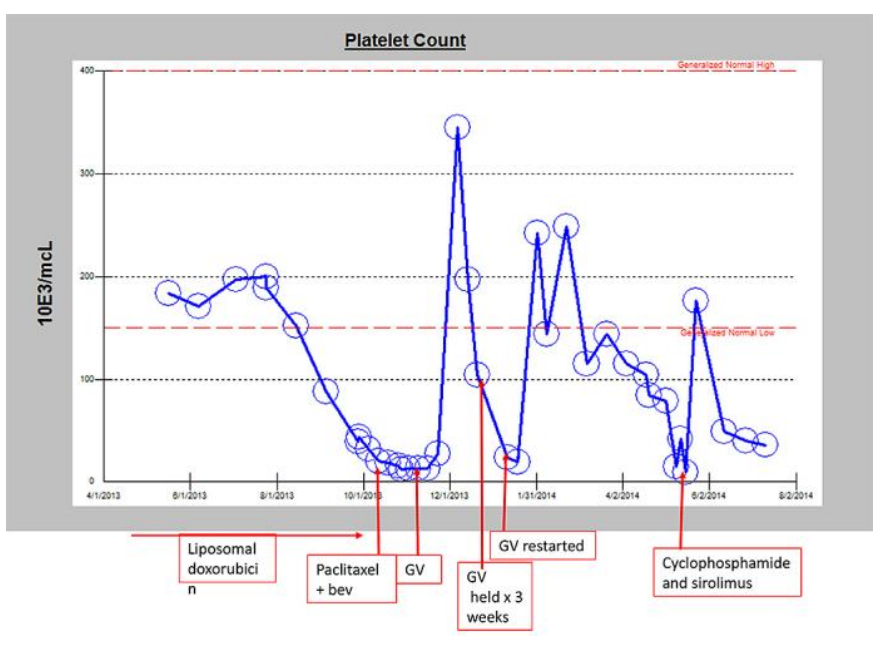

Fig. 3. Platelet trend over disease course. 